\title{
Analysis of GRACE satellite measurements over seismically active areas of the strongest earthquakes
}

\author{
Konstantin V. Simonov ${ }^{1, *}$, Valentin B. Kashkin ${ }^{2}$, Tatyana V. Rubleva ${ }^{2}$, and \\ Konstantin V. Krasnoshekov ${ }^{1}$ \\ ${ }^{1}$ Institute of Computational Modeling SB RAS, Krasnoyarsk, 660036, Akademgorodok, Russian \\ Federation \\ ${ }^{2}$ The Institute of Engineering Physics and Radioelectronics of Siberian Federal University, \\ Krasnoyarsk, 660074, Akademika Kirenskogo Str. 28/12B, Russian Federation
}

\begin{abstract}
The study is devoted to the analysis of the features of the change in the parameter EWH (Equivalent Water Height) over the geoid from the satellite measurements of the GRACE space system in seismically active areas of the strongest underwater earthquakes. The GRACE satellite data archive was created. An interpretation of the disturbed state of the geomedium using digital maps of the spatial distribution of the parameter EWH is performed.
\end{abstract}

\section{Introduction}

Methods for studying the gravitational field from satellite measurements were developed more than 40 years ago. However, only in recent years in modern space gravity missions such as CHAMP (Challenging Mini-Satellite Payload, DLR, 2000-2010), GRACE (Gravity Recovery and Climate Experiment, NASA / DLR, 2002-2017) and GOCE ( Gravity Field and Steady-State Ocean Circulation Explore, ESA, 2009-2013) continuous GPS tracking for low-orbit satellites has been implemented [1].

With the help of the CHAMP, GRACE, and GOCE systems data on the Earth's gravitational field were obtained with high accuracy and high spatial resolution. Thus, the accelerometer of the CHAMP satellite had an accuracy of $3 * 10^{-9} \mathrm{~m} / \mathrm{s}^{2}$, which is an order of magnitude smaller than the accuracy of the GRACE device equal to $1 * 10^{-10} \mathrm{~m} / \mathrm{s}^{2}$ [2]. The archive of data obtained with the help of GOCE satellite equipment contains data for only 4 years, while the GRACE information archive contains satellite measurements for 15 years, including data on space-time variations of water reserves.

Note that in the GRACE space project satellite-satellite technology is implemented using the low-low scheme, the essence of which is to measure distances and rates of change in the distance between them. Two identical GRACE satellites move 170-240 km one after another along a circular circumpolar orbit with an inclination of $89^{\circ}$ [1]. The altitude of this

\footnotetext{
* Corresponding author: simonovkv@icm.krasn.ru
} 
orbit is about $450 \mathrm{~km}$. The orbital plane of each satellite is affected by disturbing accelerations, which correspond to the first derivatives of the gravitational potential.

The equipment of GRACE satellites consists of: a highly stable frequency generator, a K-band Ranging system, a GPS receiver, an astroblock, corner reflectors for laser location, 2 accelerometers ACC and SuperSTAR. The accuracy of measuring the distance between GRACE satellites is determined by rangefinders up to $10 \mu \mathrm{m}$, which allows obtaining data on variations in gravitational force on the basis of differential measurement methods in systems with variable geometry of the arrangement of elements [3]. Satellites fly over a certain section of the Earth once every 30 days, providing a complete mapping of the earth's surface.

In this paper we studied the features of the variations of the equivalent water level (parameter EWH) over the geoid contour from the satellite measurements of the GRACE space system in the seismically active regions of the strongest earthquakes in order to reveal the connection between the stressed-deformed state of the geomedium and the processes of preparing the expected main seismic event.

The strongest underwater earthquakes with magnitudes [4] were chosen as objects of research: $\mathrm{M}=9.1$ (December 26, 2004, near Sumatra Island); $\mathrm{M}=8.1$ and $\mathrm{M}=8.3$ (November 15, 2006 and January 13, 2007, in the Simushir region); M = 8.8 (January 27, 2010, near the coast of Chile), $M=9.1$ (March 11, 2011, near the east coast of Honshu Island) and $\mathrm{M}=8.3$ (on May 24, 2013, near the Kamchatka Peninsula in Sea of Okhotsk).

\section{The gravitational field and the parameter EWH}

Satellite measurements of GRACE COP of the water surface height with respect to the geoid contour allow determining the deviation of the free surface of the sea from its average level along the satellite path. A geoid is an equipotential gravity field, which corresponds to the mean sea level at rest [5]. Monthly data obtained from the site [6] contain information on the deviation of the surface of the World Ocean from the geoid in units of the equivalent water level EWH (in cm).

In general, the parameter EWH is used to analyze the dynamic topography of the oceans and refine the parameters of the Earth's external gravitational field [7]. The method of decomposition of the Earth's gravitational potential with respect to spherical functions is described in $[5,3]$. This method of representing potential is used in the study of the gravitational field by the disturbed motion of satellites. The redistribution of water in the hydrosphere takes place in a thin layer near the surface of the Earth, just a few kilometers wide. The masses between the geoid and the water surface have a constant density $\rho_{w}$ of $1000 \mathrm{~kg} / \mathrm{m}^{3}$ [8].

The EWH is calculated on the basis of the harmonic coefficients of geopotential models minus the coefficients of the middle model (EIGEN-6C) [7]. This is a global model, the initial data of which are ground gravimetric measurements, laser trajectory observations of LAGEOS satellites (1985-2010), as well as satellite information of GOCE and GRACE. In the high-power EIGEN-6C model, the geopotential decomposition occurs to a degree of the order of 1420, which corresponds to a 14-km spatial resolution [9].

Equivalent water level (parameter EWH) is found from the ratio of surface density $\Delta \sigma$ to water density $\rho_{w}$ :

$$
E W H=\Delta \sigma / \rho_{w}
$$




\section{Results analysis}

Based on the GRACE satellite data [6], digital maps and three-dimensional images of the space-time distribution of the parameter EWH with a period of 30 days are constructed. An example of the map obtained in the isolines for December 2004 for the Sumatra earthquake region is shown in Figure 1, $a$. Figure 1, $b$ shows an image of the focal region of this earthquake in the form of a 3D model for February 2005. Figure 1, $a$ and Figure 1, $b$ the scale of the parameter EWH (in $\mathrm{cm}$ ) is given. The images shown in Figure 1, obtained with the help of satellite altimetry, reflect the features of the structure of the underlying geo-environment during the catastrophic event.

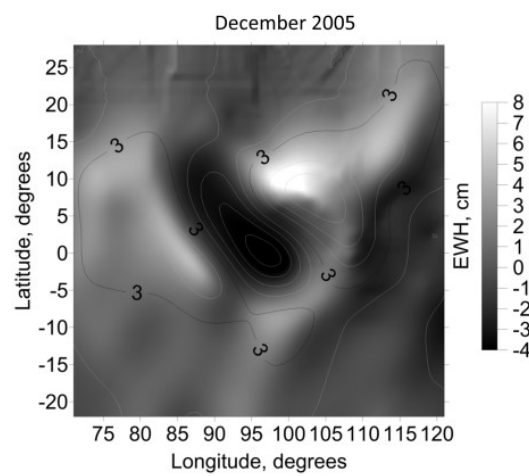

$a$

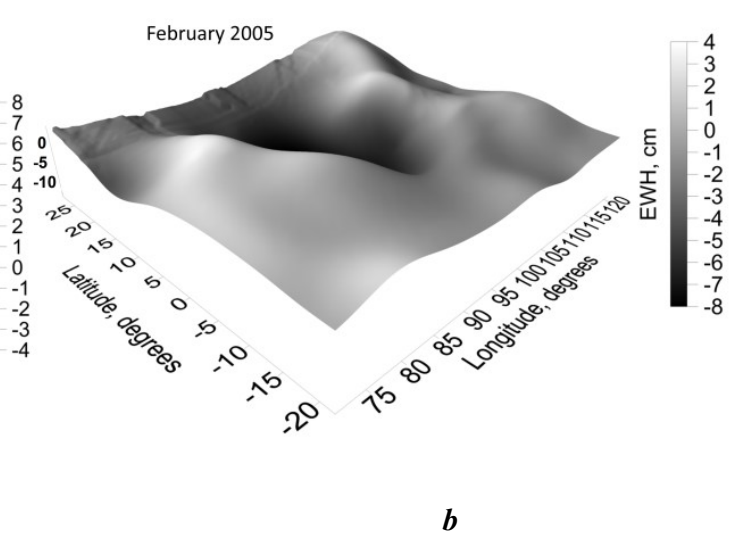

Fig. 1. Digital map in isolines for December $2004(a)$ and 3D models for February $2005(b)$ distribution of the parameter EWH of the Sumatra earthquake.

Figure 2 shows a digital map in the isolines of the spatial distribution of EWH for the seismically active region of Chile in February 2010 during the preparation and occurrence of the expected strongest earthquake according to GRACE satellite data. The point in Figure 2 indicates the hypocentre of the earthquake. You can see an abnormal area with increased values of the parameter EWH. It is revealed that the hypocenter of the earthquake is located near the anomaly axis, which is oriented submeridionally, at the maximum gradients of the parameter EWH. Interpretation of the direction of the revealed anomaly is related to the geometry of the subduction zone of lithospheric plates. 


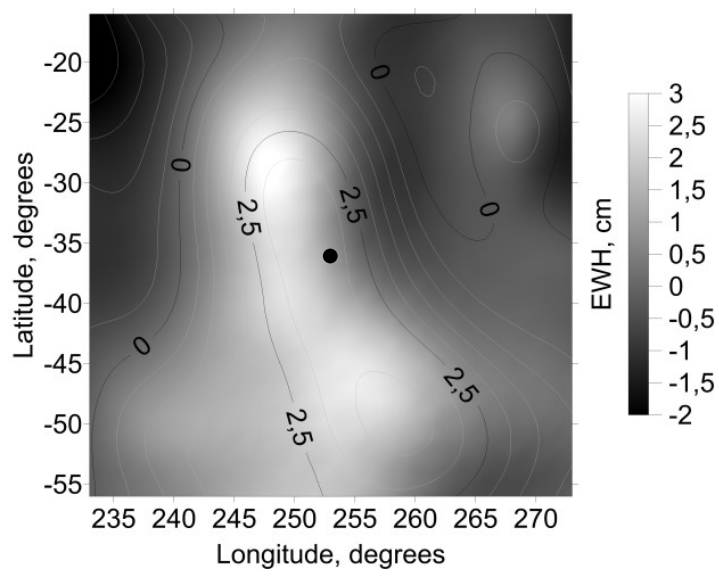

Fig. 2. Digital map in isolines of the Chilean earthquake for February 2010.

A graph of the change in EWH at the hypocentral point was plotted 5 months before the seismic event and 5 months after the earthquake $\mathrm{Mw}=8.8$ (Figure 3). The characteristic change in the parameters EWH, shown in Figure 3, shows the process of preparing the strongest earthquake and its aftershock discharge. For " 0 " on the horizontal time scale in Figure 3 the date of the considered seismic event is adopted. The "negative part" of this scale characterizes the process of preparing the strongest earthquake, the "positive part" is aftershock activity. The vertical scale in Figure 3 reflects the changes in the parameter EWH, characterizing the sharp increase in the water surface boundary during the process of making. Dedication is understood as the decompression of the upper layers of the geomedia and their saturation with water.

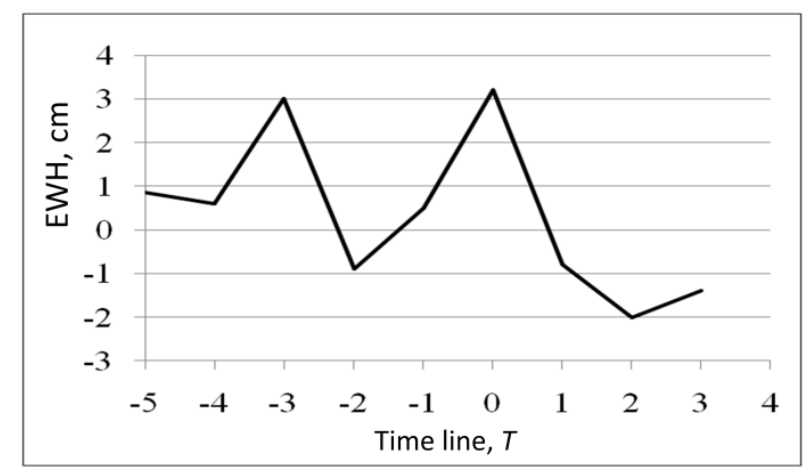

Fig. 3. The dynamics of the change in the parameter EWH with a period of $1 T=30$ days during the process of preparing the strongest earthquake and its aftershock discharge.

With a deep earthquake (Sea of Okhotsk, May 2013), a zone of increased EWH values was observed in the area of preparation (Figure 4). The hypocenter of the earthquake was located at a depth of $602 \mathrm{~km}$ and Figure 4 it is indicated by a dot. The digital map shows that the anomalous region with increased values of the parameter EWH is oriented in the SW-NE direction. From Figure 4 it becomes clear that the hypocenter of the earthquake is located near the axis of the anomaly at the maximum gradients of the parameter EWH. It is important to note that, in contrast to subduction earthquakes, which have a pronounced linear geostructure, a deep event is characterized by a localized focal zone. 


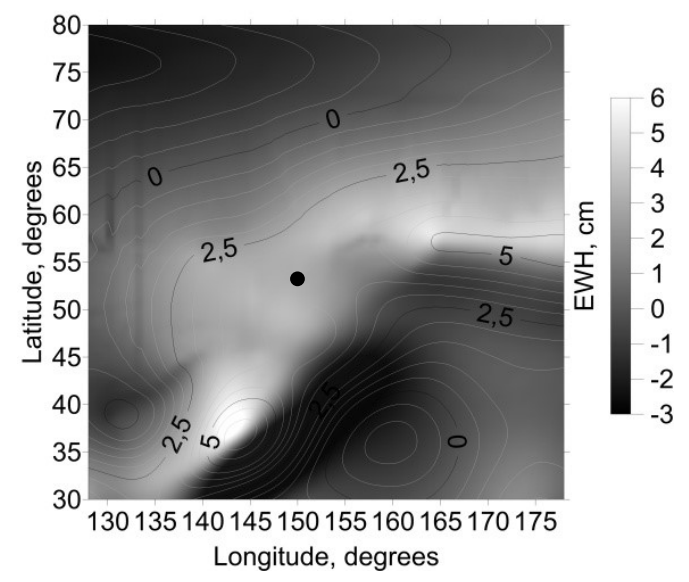

Fig. 4. Digital map in isolines of the Okhotsk earthquake for May 2013.

\section{Conclusion}

As a result of the research, a GRACE satellite measurement archive has been compiled containing information parameter data on the EWH (equivalent water level). The parameter EWH during the preparation and relaxation of the strongest earthquakes varies over the contour of the geoid. GRACE data have been analyzed for a number of areas of focal zones of known underwater earthquakes (2004-2013). Digital maps of the EWH variation over the geoid in the focal zones of the strongest earthquakes studied are constructed. The specified parameter can serve as a basis for an estimation of geodynamic risks of occurrence of destructive earthquakes for coastal seismically dangerous territories.

\section{References}

1. F. Flechtner, N. Sneeuw, Wolf-Dieter Schuh (Eds.), Science Report. Advanced Technologies in Earth Sciences, Springer, 20 (2014)

2. I.N. Gansvind, Electronic Libraries, 20, 1 (2017)

3. R.A. Kashcheev, Modern methods of satellite gravimetry (Kazan. Univ., 2015)

4. USGS, Latest Earthguakes, https://earthquake.usgs.gov/earthquakes/map

5. B. Hoffmann-Vellenhof, G. Moritz, Physical geodesy (Moscow, MIIGAiK, 2007)

6. NASA, ftp PO.DAAC, ftp://podaac-ftp.jpl.nasa.gov/allData/tellus/L3/ocean_mass /RL05/ascii/

7. B. Wouters, J.A. Bonin, D.P. Chambers, R.E.M. Riva, I. Sasgen, J. Wahr, Review Article Rep., Prog. Phys. (2014)

8. J. Wahr, M. Molenaar, F. Bryan, J. Geoph. Res., Solid Earth, 103, B 12 (1998)

9. V.F. Kanushin, A.P. Karpik, I.G. Ganagina, D.N. Golodobin, A.M. Kosareva, N.S. Kosarev, Investigation of modern global models of the Earth's gravitational field, (Novosibirsk, SSUGiT, 2015) 